EPJ manuscript No.

(will be inserted by the editor)

\title{
Phase behavior of three-component ionic fluids
}

\author{
André G. Moreira ${ }^{a}$ and Roland R. Netz \\ Max-Planck-Institut für Kolloid- und Grenzflächenforschung, 14424 Potsdam, Germany \\ Received: date / Revised version: date
}

\begin{abstract}
We study the phase behavior of solutions consisting of positive and negative ions of valence $z$ to which a third ionic species of valence $Z>z$ is added. Using a discretized Debye-Hückel theory, we analyze the phase behavior of such systems for different values of the ratio $\bar{Z} \equiv Z / z$. We find, for $\bar{Z}>1.934$, a three-phase coexistence region and, for $\bar{Z}>2$, a closed (reentrant) coexistence loop at high temperatures. We characterize the behavior of these ternary ionic mixtures as function of charge asymmetry and temperature, and show the complete phase diagrams for the experimentally relevant cases of $\bar{Z}=2$ and $\bar{Z}=3$, corresponding to addition of divalent and trivalent ions to monovalent ionic fluids, respectively.
\end{abstract}

PACS. 61.20.Qg Structure of associated liquids: electrolytes, molten salts, etc. - 52.25.Kn Thermodynamics of plasmas - 05.20.-y Classical statistical mechanics

\section{Introduction}

In many areas of Physics, Chemistry, and Biology electrostatic forces play an important role in determining system properties. This becomes particularly true when dealing with ionic fluids, i.e., fluids consisting of dissociated cations and anions, since in most cases the Coulomb interaction is the dominant interaction between the particles. Ionic mixtures have been intensely debated not only because of their interesting behavior (for recent reviews, see [1, 2, 3, 4, 5]), but also because a better understanding of ' such systems is the first step towards a more rigorous theoretical treatment of more complicated systems like charged colloids and polyelectrolytes.

It has been shown experimentally that ionic solutions exhibit liquid-liquid phase coexistence, terminated by a critical point, similar to ordinary (nonionic) fluids 6]. Theoretically, such a demixing transition has been rationalized in terms of an effective attraction between the ions [7, 8 which is due to charge screening, first introduced by Debye and Hückel[9]. For simple inorganic salts, such as $\mathrm{NaCl}$, this critical point occurs at temperatures above $3000 \mathrm{~K}$ [6] and thus precludes precise measurements. More detailed experiments became available with the advent of large organic ions, which show critical points at temperatures of $414 \mathrm{~K} 10$ and, more recently, at $317 \mathrm{~K} 11$.

The aforementioned examples correspond to binary mixtures of cations and anions of the same valence. In this article we will look at three-component ionic mixtures, i.e., we will consider solutions of $z: z$ salts to which a third component with valence $Z$ is added (keeping total charge neutrality). This situation often occurs in real

\footnotetext{
a e-mail: amoreira@mpikg-golm.mpg.de
}

systems, deserving for this reason a closer look. For instance, in the cellular medium several ionic species with different valences (like $\mathrm{Cl}^{-}, \mathrm{Na}^{+}$and $\mathrm{Ca}^{2+}$ ) are simultaneously present. As another example, the presence of charged impurities can change dramatically the behavior of charge-stabilized colloidal suspensions 12. With this in mind, adding a third component to an ionic fluid is the natural extension of previous work and, as we will show, the resulting phase behavior is quite rich: it is our hope that this will motivate experiments testing our predictions.

The theoretical tools that are usually employed to treat ionic fluids are the Debye-Hückel theory [9] (or some modified version of it) and liquid state theory with some special approximation 13 like the hypernetted chain (HNC) or the mean-spherical approximation (MSA). For instance, Caccamo 14 and Caccamo and Malescio 15 studied ternary mixtures with $\bar{Z} \equiv Z / z=2$, the former using MSA (for low-density mixtures), while the latter made a comparison between MSA, HNC and Monte Carlo results (at high salt densities). Also, three-component mixtures with $\bar{Z}=2$ and 3 have been studied by Kenkare et al. 116., where MSA was used to compute the Coulombic contribution to the free energy. Their phase diagrams, although presented in a constant-pressure ensemble (in contrast to our phase diagrams, where we do not constrain the osmotic pressure to constant values), seem to be generally in accordance with our results 17, 18.

In general, the comparison between computer simulations and liquid state theory shows that the latter leads to quite accurate predictions for the thermodynamic variables of the system. The same cannot be said about the Debye-Hückel theory (cf. Table 1): although it should be 
André G. Moreira and Roland R. Netz: Phase behavior of three-component ionic fluids

exact in the limit of vanishing densities, it quickly deviates from the results obtained by computer simulations as one increases the salt density. However, if one is interested in low-density systems, Debye-Hückel has the advantage of offering a very simple free energy that, although not very accurate, can still be used to study the phase behavior of ionic systems without considerable numerical work (in contrast to most approximations used in liquid state theory). Besides, Debye-Hückel has been shown 19,20 to be a Gaussian theory that can be improved, with the help of field-theoretic methods, through a systematic expansion of the free energy in cumulants of a fluctuating field (which can be related to the electrostatic potential). For these reasons, we will use here the free energy derived from the Debye-Hückel theory to describe our system.

Our article is presented as follows: in section 2 we briefly introduce the theoretical background of our work, in section 3 we present the resulting phase diagrams for various three-component systems and fully characterize their phase behavior as a function of $\bar{Z}$ and temperature and finally section 4 contains some concluding remarks.

\section{Theoretical background}

Let us assume a symmetric ionic system with cations and anions of valence $z$ to which a third ionic species of valence $Z$ is added. Let us also assume that the ions interact only via the Coulomb force, or that any solvophobic interaction between the ions are weak when compared to the Coulomb interaction. In this case, following what has been derived in Ref. [19], the Debye-Hückel contribution to the bulk free energy per volume is

$$
f_{\mathrm{DH}}=-\int \frac{\mathrm{d} \mathbf{q}}{(2 \pi)^{3}}\left[\frac{\kappa^{2}}{2 q^{2}}-\frac{1}{2} \ln \left(1+\frac{\kappa^{2}}{q^{2}}\right)\right]
$$

where the electrostatic self-energy is subtracted and the momentum integral goes over a cube of length $2 \pi / a$. This accounts for the lattice (with spacing $a$ ), implementing in an approximate way the ionic hard cores. The screening length $\kappa^{-1}$ is defined by

$$
\kappa^{2}=4 \pi \ell_{B}\left(z^{2} c_{+}+z^{2} c_{-}+Z^{2} c\right)
$$

where the concentrations of the positive and negative ions of valence $z$ are denoted by $c_{+}$and $c_{-}$, and the concentration of the third component (with valence $Z$ ) is denoted by $c$. The Bjerrum length $\ell_{B} \equiv e^{2} /\left(4 \pi \varepsilon k_{B} T\right)$ (in SI units) is the distance at which the electrostatic energy between two elementary charges equals the thermal energy $k_{B} T$.

Since the integrand in Eq. (11) is isotropic, we distort the integration volume to a sphere and obtain after a straightforward integration

$f_{\mathrm{DH}}=-\frac{\kappa^{3}}{6 \pi^{2}} \arctan \left[\frac{\pi}{a \kappa}\right]-\frac{\kappa^{2}}{12 \pi a}+\frac{\pi}{12 a^{3}} \ln \left[1+\frac{a^{2} \kappa^{2}}{\pi^{2}}\right]$.

In the limit $a \rightarrow 0$ this reduces to $f_{\mathrm{DH}} \simeq-\kappa^{3} / 12 \pi$, which is the well-known Debye-Hückel limiting law. The full (Helmholtz) free energy density contains also the ideal entropy of mixing and reads

$$
f=c \ln c+c_{+} \ln c_{+}+c_{-} \ln c_{-}+f_{\mathrm{DH}} .
$$

Global charge neutrality implies that $Z c+z c_{+}=z c_{-}$, where, without loss of generality, the third component of valence $Z$ is assumed to be positively charged. This allows the elimination of one of the concentrations from the free energy in Eq. (4): in the following, we choose to eliminate $c_{-}$. For representing our results, we introduce the usual rescaled temperature $T^{*} \equiv a / \ell_{B} z^{2}$ and the volume fractions $\phi^{+} \equiv a^{3} c_{+}$and $\phi \equiv a^{3} c$ (notice that the usual definition of volume fraction - normally represented as $\eta$-is related to our definition through $\phi=6 \eta / \pi)$.

If one of the three species is absent, the system reduces to a two-component system, which has been studied (using the same free energy) in Ref. [19]. If $\phi=0$, we obtain the lower limiting critical temperature at $T_{\mathrm{LLC}}^{*}=0.1776$ with a critical density of positive ions of $\phi_{\mathrm{LLC}}^{+}=0.0209$; if $\phi^{+}=0$, we obtain the upper limiting critical temperature at $T_{\mathrm{ULC}}^{*}=0.1776 \bar{Z}$ and $\phi_{\mathrm{ULC}}=0.0418 /(1+\bar{Z})$. Clearly, since $\bar{Z}>1$, we have $T_{\mathrm{ULC}}^{*}>T_{\mathrm{LLC}}^{*}$. These temperatures will be used as "guidelines" in the presentation of the phase behavior of three-component systems.

In order to obtain the phase diagrams, we calculate the chemical potentials $\mu$ and $\mu^{+}$from the free energy Eq. (4) by taking derivatives with respect to $c$ and $c_{+}$, respectively. We also calculate the osmotic pressure $p$, which can be easily shown to be $p=-f+c \mu+c_{+} \mu^{+}$(in units of $\left.k_{B} T\right)$. We look for instabilities in the free energy through the sign of the determinant of the (symmetric) matrix

$$
J=\left(\begin{array}{cc}
\frac{\partial^{2} f}{\partial c^{2}} & \frac{\partial^{2} f}{\partial c \partial c_{+}} \\
\frac{\partial^{2} f}{\partial c_{+} \partial c} & \frac{\partial^{2} f}{\partial c_{+}^{2}}
\end{array}\right)
$$

in the plane $\left(c, c_{+}\right)$(or equivalently in the plane $\left(\phi, \phi^{+}\right)$) at fixed values of $T^{*}$. The global convexity of the free energy implies that $\operatorname{det} J$ should be always positive: when this is not verified, one is in a region where phase separation occurs. The coexisting phases are then found through the Maxwell construction 21], i.e., one has to find the $c$ and $c_{+}$ in each of the coexisting phases that lead to values of $\mu$, $\mu^{+}$and $p$ that are same for all phases in coexistence. This procedure yields the phase diagrams that we now present.

\section{Phase diagrams}

In Fig. 1 we present the phase behavior for $\bar{Z}=1.8$, representative of small charge ratios $\bar{Z}<1.934$. For $T^{*}>$ $T_{\mathrm{ULC}}^{*}=0.3196$ no phase separation occurs. In Fig. 1a we show, to the left, the phase diagram in fugacities (which are defined as the exponential of the chemical potentials) and, to the right, the phase diagram in volume fractions for $T^{*}=0.2500$, representative of the range $T_{\mathrm{LLC}}^{*}<T^{*}<$ $T_{\mathrm{ULC}}^{*}$. There is one critical point at the terminus of a phase coexistence line which emanates from the axis defined by $\phi^{+}=0\left(\right.$ or $\left.\mathrm{e}^{\mu^{+}}=0\right)$. At $T^{*}=T_{\mathrm{LLC}}^{*}$ this critical point hits 




Fig. 1. Phase diagrams at constant reduced temperature $T^{*} \equiv a / \ell_{B} z^{2}$ for a three-component system with a valence ratio $\bar{Z}=1.8$-representative of systems with $\bar{Z}=Z / z<1.934$-as function of $\mathrm{e}^{\mu}$ and $\mathrm{e}^{\mu^{+}}$(the fugacities of $Z$-valent ions and of positive $z$-valent ions, respectively) and as function of $\phi \equiv a^{3} c$ and $\phi^{+} \equiv a^{3} c_{+}$(the volume fractions of $Z$-valent ions and of positive $z$-valent ions). Filled circles denote critical points. Plots: (a) $T^{*} \equiv a / \ell_{B} z^{2}=0.2500$ (representative of the temperature range $T_{\mathrm{LLC}}^{*}<T^{*}<T_{\mathrm{ULC}}^{*}$ ); (b) $T^{*}=0.1754$ (representative for $T^{*}<T_{\mathrm{LLC}}^{*}$ ).

the axis $\phi=0$ (or $\left.\mathrm{e}^{\mu}=0\right)$ at $\phi_{\mathrm{LLC}}^{+}$, and for lower temperatures there is single coexistence line connecting the axes $\phi^{+}=0$ and $\phi=0$. This is demonstrated in Fig. 1b, where we show a phase diagram for $T^{*}=0.1754$.

The behavior is dramatically altered for larger charge asymmetry, viz. $\bar{Z}>1.934$. One important difference in the phase topology is that such systems always have two critical end-points, one at temperature $T_{\mathrm{UCEP}}^{*}$ (upper critical end-point) and another one at $T_{\mathrm{LCEP}}^{*}$ (lower critical end-point). In the temperature range between these two critical end-points the systems exhibit three phase coexistence.

In Fig. 2 we present the phase behavior for $\bar{Z}=2$ corresponding, for instance, to a mixture of a divalent cations with monovalent co- and counter-ions. For high temperatures, $T^{*}>T_{\mathrm{ULC}}^{*}=0.3552$ no phase separation occurs. For a temperature range $T_{\mathrm{UCEP}}^{*}<T^{*}<T_{\mathrm{ULC}}^{*}$ there is a single critical point, as demonstrated in Fig. 2a for $T^{*}=0.2070$; the phase diagram is similar to the one shown in Fig. 1a. The upper critical end-point is located at $T_{\mathrm{UCEP}}^{*}=0.1808$, below which a second critical point exists. The resulting phase behavior for $T_{\mathrm{LCEP}}^{*}<T^{*}<$ $T_{\mathrm{UCEP}}^{*}$ is similar to Fig. $2 \mathrm{~b}$, which depicts the phase diagram for $T^{*}=0.1800$, and where we see two phase coexistence lines, each terminating at a critical point and merging at a triple point. The region around this triple point is highlighted at the phase diagram in volume fractions (Fig. 2b, right). At $T^{*}=T_{\mathrm{LCEP}}^{*}=0.1796$ one of

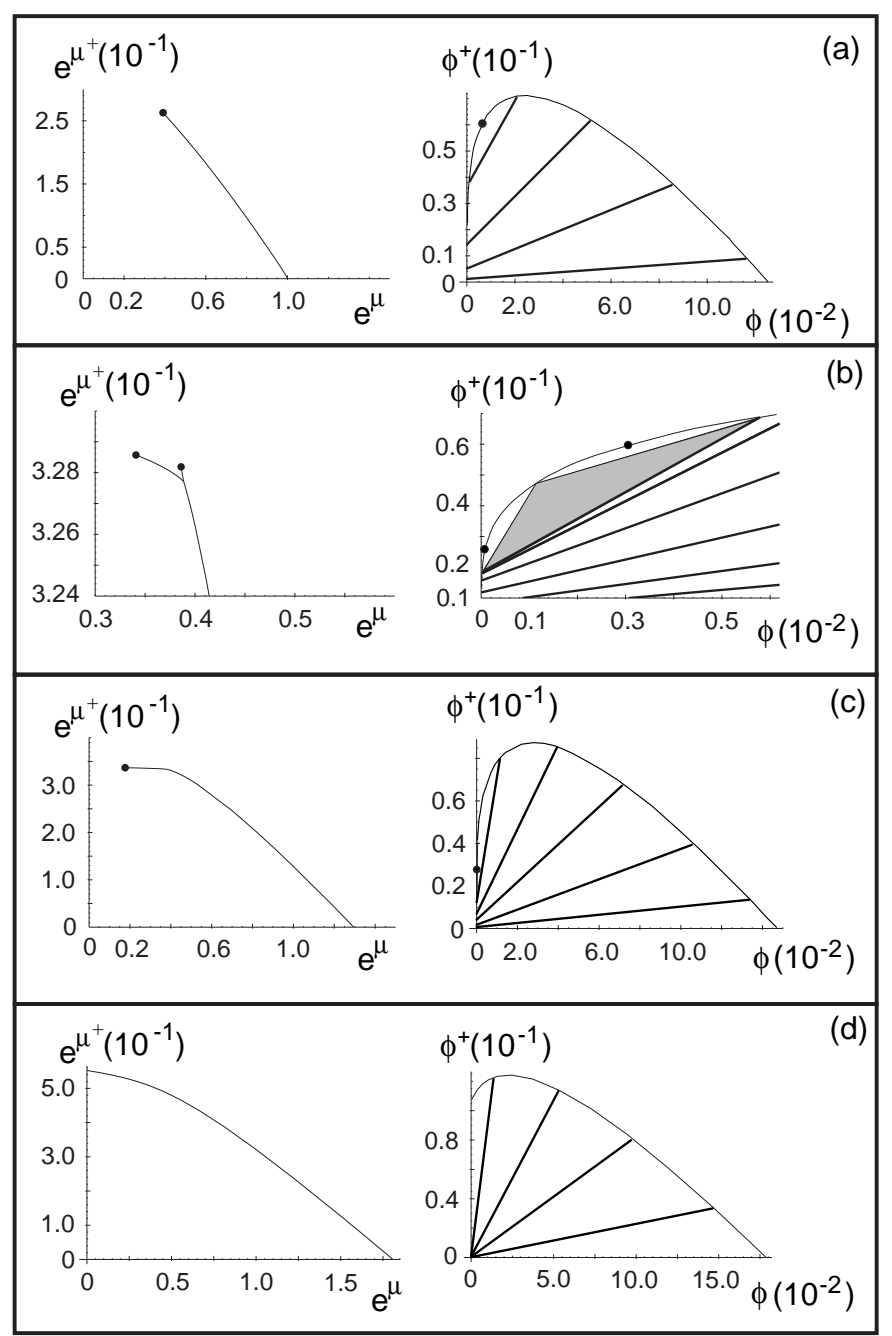

Fig. 2. Phase diagrams at constant $T^{*} \equiv a / \ell_{B} z^{2}$ for $\bar{Z}=2$. The notation is the same as in Fig. 1. Shaded areas in the volume fraction representation denote three phase coexistence zones. Plots: (a) $T^{*}=0.2070\left(T_{\mathrm{UCEP}}^{*}<T^{*}<T_{\mathrm{ULC}}^{*}\right)$; (b) $T^{*}=0.1800\left(T_{\mathrm{LCEP}}^{*}<T^{*}<T_{\mathrm{UCEP}}^{*}\right)$ (only shown close to the three phase coexistence zone); (c) $T^{*}=0.1786\left(T_{\mathrm{LLC}}^{*}<T^{*}<\right.$ $\left.T_{\mathrm{LCEP}}^{*}\right) ;(\mathrm{d}) T^{*}=0.1429\left(T^{*}<T_{\mathrm{LLC}}^{*}\right)$.

the critical lines present for $T_{\mathrm{LCEP}}^{*}<T^{*}<T_{\mathrm{UCEP}}^{*}$ terminates at the lower critical end-point. For $T_{\mathrm{LLC}}^{*}<T^{*}<$ $T_{\mathrm{LCEP}}^{*}$ we are thus left again with a single critical point; a representative phase diagram is shown in Fig. 2c for $T^{*}=0.1786$. Finally, for $T^{*}<T_{\mathrm{LLC}}^{*}=0.1776$, no critical point is observed; Fig. 2d shows such a phase diagram for $T^{*}=0.1429$, which is similar to the one in Fig. $1 \mathrm{~b}$.

The complete phase behavior is summarized in Fig. 3, where we plot the various critical temperatures as a function of the valence ratio $\bar{Z}$. In Fig. 3a we show, in the vicinity of $\bar{Z}=2$, the upper critical end-point temperature $T_{\mathrm{UCEP}}^{*}$ (broken line), the lower critical end-point temperature $T_{\mathrm{LCEP}}^{*}$ (dotted-broken line), and the lower limiting critical temperature $T_{\mathrm{LLC}}^{*}$ (solid line). The two lines of critical end-points meet at a double critical end-point at 


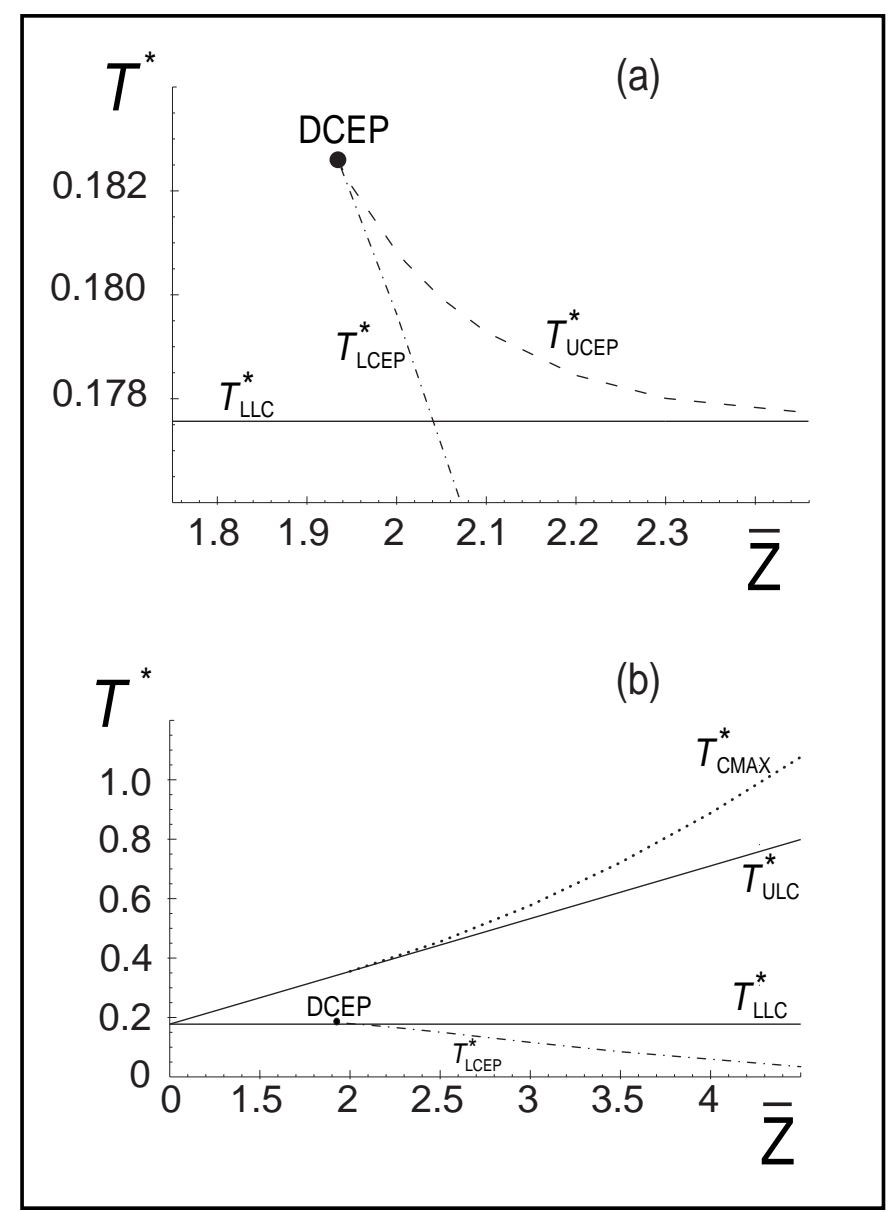

Fig. 3. (a) Plot of the upper critical end-point temperature $T_{\mathrm{UCEP}}^{*}$ (broken line), the lower critical end-point temperature $T_{\mathrm{LCEP}}^{*}$ (dotted-broken line), and the lower limiting critical temperature $T_{\mathrm{LLC}}^{*}$ (solid line) as a function of the valence ratio $\bar{Z}$ in the vicinity of the double critical end-point (DCEP). (b) Plot of the lower and upper limiting critical temperature, the maximal critical temperature $\left(T_{\text {CMAX }}^{*}\right)$ and the lower critical end-point temperature as a function of $\bar{Z}$; notice the widening of the three phase zone and the growth of $T_{\text {CMAX }}^{*}$ as $\bar{Z}$ increases.

$\bar{Z}=1.934$, below which no triple point occurs. On a larger scale (Fig. 3b) it becomes clear that the triple phase temperature range widens as $\bar{Z}$ increases (note that $T_{\mathrm{UCEP}}^{*}$ and the double critical end-point cannot be distinguished from $T_{\mathrm{LLC}}^{*}$ at this scale). We also plot the maximal temperature at which a critical point occurs, $T_{\text {CMAX }}^{*}$, which for $\bar{Z}>2$ satisfies $T_{\mathrm{CMAX}}^{*}>T_{\mathrm{ULC}}^{*}$. This leads to closed coexistence loops, as is demonstrated in the following.

In Fig. 4 we show the phase behavior for $\bar{Z}=3$. In Fig. 4a we plot the phase diagram for $T^{*}=0.5747$, which is below the maximal critical temperature $T_{\mathrm{CMAX}}^{*}=$ 0.5771 and above the upper limiting critical temperature $T_{\mathrm{ULC}}^{*}=0.5328$. Notice the presence of two critical points bounding a closed coexistence loop. For $T_{\mathrm{UCEP}}^{*}<T^{*}<$ $T_{\mathrm{ULC}}^{*}$ we find a phase topology with a single critical point, as depicted in Fig. 4b for $T^{*}=0.5155$. In this system the upper critical end-point occurs at a temperature very close to the lower limit critical temperature (this is already clear in Fig. 3); more precisely, $T_{\mathrm{LLC}}^{*}=0.1775656$ and $T_{\mathrm{UCEP}}^{*}=$ $T_{\mathrm{LLC}}^{*}+1.0 \times 10^{-6}$. In Fig. $4 \mathrm{c}$, where $T^{*}=T_{\mathrm{LLC}}^{*}+0.7 \times 10^{-6}$ $\left(T_{\mathrm{LLC}}^{*}<T^{*}<T_{\mathrm{UCEP}}^{*}\right)$, we observe a triple point and two critical points. Since the lower critical end-point occurs at a lower temperature than the lower limiting critical point (cf. Fig. 3), we obtain in the range $T_{\mathrm{LCEP}}^{*}<T^{*}<T_{\mathrm{LLC}}^{*}$ one critical point and a triple point, as shown in Fig. $4 \mathrm{~d}$ for $T^{*}=0.1667$. Finally, for $T^{*}<T_{\mathrm{LCEP}}^{*}=0.1163$, all critical points have disappeared and a coexistence line connects the two phase diagram axes, as shown in Fig. 4e for $T^{*}=0.1000$.

\section{Conclusion}

For small valence ratios $(\bar{Z} \equiv Z / z<1.934)$ we have shown that the the two critical points obtained for the limiting cases (i.e., one of the two positively charged species is absent) are joined by a continuous critical line. For larger valence ratios $(\bar{Z}>1.934)$ we have obtained more complicated phase diagrams, with a line of triple points bounded by critical end-points and closed coexistence rings at $T>$ $T_{\mathrm{ULC}}^{*}$ for $\bar{Z}>2$. We have also made a global analysis of three-component ionic mixtures, where their phase behavior is characterized as a function of charge asymmetry (up to $\bar{Z} \sim 5$ ) and temperature.

Denoting by $T_{\mathrm{LLC}}^{*}$ the lower limiting critical temperature of the symmetric ionic system, we have shown that this triple line extends, for $\bar{Z}=2$, over a temperature window of $1.012 T_{\mathrm{LLC}}^{*}<T^{*}<1.018 T_{\mathrm{LLC}}^{*}$ and, for $\bar{Z}=3$, over $0.66 T_{\mathrm{LLC}}^{*}<T^{*} \lesssim T_{\mathrm{LLC}}^{*}$. This means that for a $1: 1$ organic salt with a critical point at $T \simeq 400 \mathrm{~K}$ this temperature window would have a $2.4 \mathrm{~K}$ width when adding divalent ions and a $136 \mathrm{~K}$ width when adding trivalent ions. The triple line should in principle be observable with a suitable choice of mono-, di- and trivalent organic ions. However, one should be careful when examining the numbers presented here. Although the Debye-Hückel theory successfully predicts for ionic systems the existence of a liquid-liquid critical point and the high asymmetry of their phase diagrams, it has some limitations, as we now discuss.

One problem is the fact that the theory is quite sensitive to the way that the hard cores are treated: we chose to include them through a lattice of spacing $a$. Alternatively, one could add to the free energy Eq. (位) an extra term to account for the hard-core repulsion (for instance a free-volume approximation term) and get the electrostatic contribution $f_{\mathrm{DH}}$ either by integrating Eq. (1i) without the cut-off (which results immediately in the Debye-Hückel limiting law) or by doing a "charging process" after solving of the linearized Poisson-Boltzmann equation with the appropriate boundary conditions for the hard cores 9.22 , 23]. These methods yield similar phase diagrams, but with different values of temperature and density for the critical point. The use of a lattice is the most convenient for the field-theoretic treatment, although it is certainly not the best way 24. This, as well as some simplifications done 


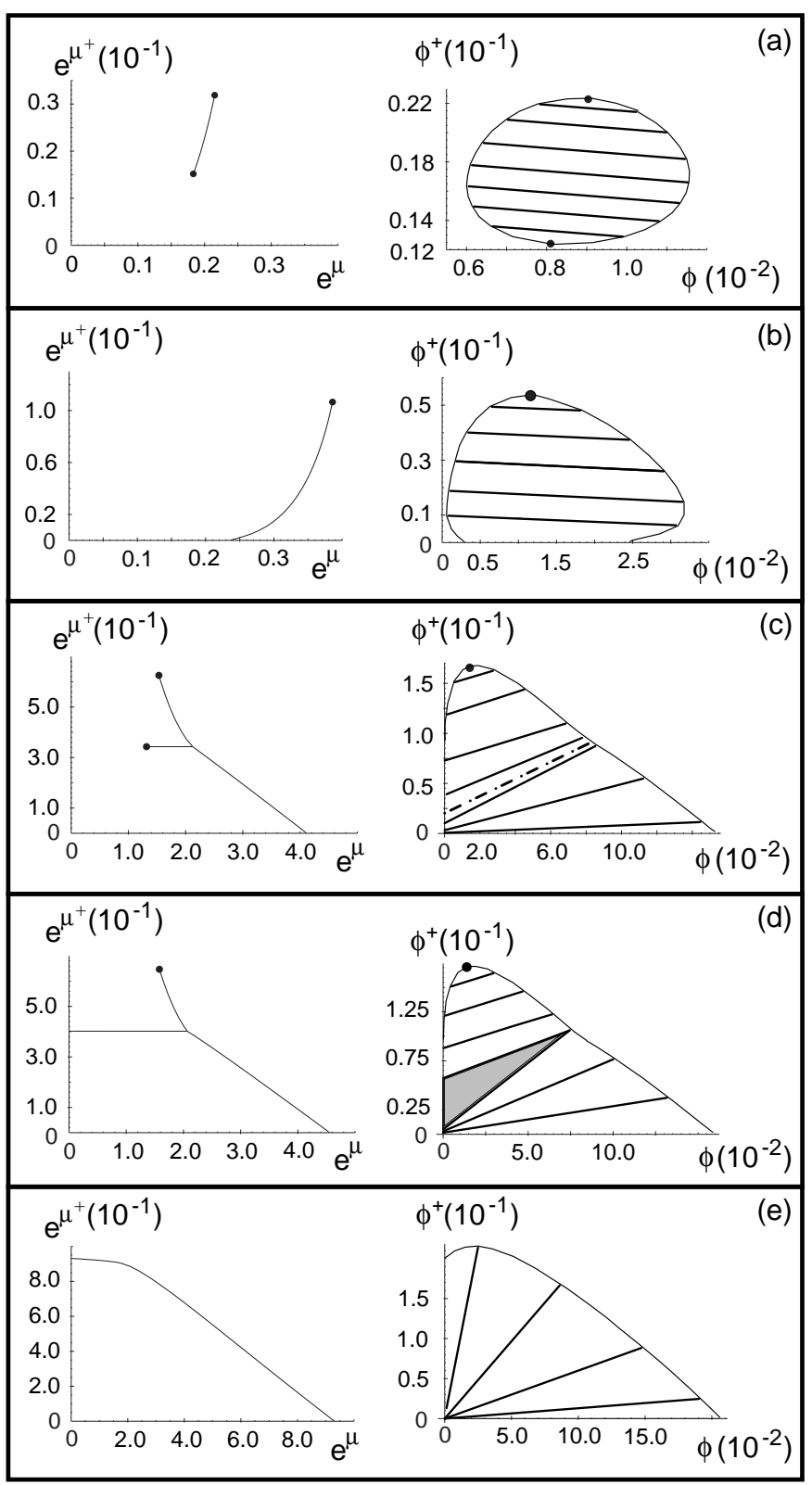

Fig. 4. Phase diagrams at constant $T^{*} \equiv a / \ell_{B} z^{2}$ for $\bar{Z}=3$. The notation is the same as in Figs. 1 and 2. Plots: (a) $T^{*}=$ $0.5747\left(T_{\mathrm{ULC}}^{*}<T^{*}<T_{\mathrm{CMAX}}^{*}\right) ;(\mathrm{b}) T^{*}=0.5155\left(T_{\mathrm{UCEP}}^{*}<\right.$ $\left.T^{*}<T_{\mathrm{ULC}}^{*}\right) ;$ (c) $T^{*}=0.1775663\left(T_{\mathrm{LLC}}^{*}<T^{*}<T_{\mathrm{UCEP}}^{*}\right)($ in volume fraction the three phase coexistence is represented by a dashed line); (d) $T^{*}=0.1667\left(T_{\mathrm{LCEP}}^{*}<T^{*}<T_{\mathrm{LLC}}^{*}\right) ;(\mathrm{e})$ $T^{*}=0.1000\left(T^{*}<T_{\mathrm{LCEP}}^{*}\right)$.

during the calculations (like performing the integral in Eq. (11) in the sphere instead of the initial cube of size $2 \pi / a)$ are expected to affect the final results.

The direct comparison of the internal energy per particle that follows from Eq. (凷), viz.

$$
u \equiv \frac{U_{\mathrm{ex}}}{N k_{B} T}=-\frac{a^{3} \kappa^{3}}{8 \pi \phi^{+}} \arctan \left(\frac{\pi}{a \kappa}\right),
$$

\begin{tabular}{cccccc}
\hline$\phi^{+}$ & MC & HNC & MSA & DH & Eq. (6) \\
\hline binary & & & & & \\
$4.2 \times 10^{-4}$ & 0.1029 & 0.1014 & 0.0992 & 0.1120 & 0.1090 \\
$4.8 \times 10^{-3}$ & 0.2739 & 0.2714 & 0.2675 & 0.3780 & 0.3438 \\
$1.9 \times 10^{-2}$ & 0.4341 & 0.4295 & 0.4264 & 0.7651 & 0.6276 \\
$4.6 \times 10^{-2}$ & 0.5516 & 0.5447 & 0.5405 & 1.1736 & 0.8610 \\
\hline ternary & & & & & \\
$2.1 \times 10^{-3}$ & - & 1.520 & 1.301 & 2.327 & 1.913 \\
\hline
\end{tabular}

Table 1. Comparison between (minus) the excess internal energy per particle from Monte Carlo simulations (MC), hypernetted chain (HNC), mean spherical approximation (MSA), Debye-Hückel limiting law (DH) and Eq. (6) $\left(\phi^{+}=a^{3} c_{+}\right.$, as defined in Section 2). In the first part of the table ("binary") the comparison is done for a simple $1: 1$ electrolyte with $T^{*}=0.59$, where the data for MC, HNC and MSA are taken from Table II of Ref. [25]. In the second part of the table ("ternary"), the same comparison is done for a three component mixture with $\bar{Z}=2, T^{*}=0.294$ and $\phi=\phi^{+}$; the data for HNC and MSA are taken from Table I of Ref. [14].

with results from other approaches is shown in Table 1 (in Eq. (6), $\kappa$ is the inverse screening length defined in Eq. (2), $a$ is the lattice spacing, corresponding to the ionic diameter, and $\phi^{+}$is the volume fraction of the positive $z=1$ ions). In the first part of this table ("binary"), we compare the values of $u$ from Monte Carlo simulations, HNC, MSA 25] and Debye-Hückel limiting law (DH) for a simple 1:1 ionic mixture (where $\phi=0$, i.e., the $Z$-valent ions are not present). Although our results are better than the pure DH results, it is clear that our theory needs improvement at higher volume fractions. In the second part of table 1 ("ternary"), the same comparison is done for a threecomponent mixture with $\bar{Z}=2$ with results obtained by Caccamo 14. (notice that the value of $\phi^{+}$is in the region where our phase diagrams are shown). This shows that our theory underestimates the value of the internal energy of the system, which indicates that, beyond the problem with the hard cores, a more refined free energy is needed in order to capture correctly all features of the ionic fluids [26]. This idea is reinforced by the discrepancy between the critical parameters for a $1: 1$ salt obtained through MC simulations 27 (which lie in the range $T_{\mathrm{C}}^{*}=0.049-0.070$ and $\left.\phi_{\mathrm{C}}^{+}=0.013-0.040\right)$ with the ones obtained with the free energy used here $\left(T_{\mathrm{C}}^{*}=0.178\right.$ and $\left.\phi_{\mathrm{C}}^{+}=0.021\right)$.

With this in mind, one should regard the results we obtained as being of "zeroth-order." As previously mentioned, the free energy used here is the lowest-order result following a systematic expansion. Changes in the phase behavior are expected as one takes into account higher order terms in the expansion, but as shown in Ref. [19] for $Z: z$ mixtures, the inclusion of terms up to the eighth cumulant in the fluctuating field affects the phase diagrams appreciably only for values of $\bar{Z} \equiv Z / z \gtrsim 5$. For this reason, and in spite of the limitations of the DebyeHückel theory, we assume that the topology of the phase diagrams shown here are correct. 
We thank P. van der Schoot for stimulating discussions and H. Weingärtner and J. M. H. Levelt Sengers for useful correspondence. AGM acknowledges the support from the Portuguese FCT through grant PRAXIS XXI/BD/13347/97.

\section{References}

1. J. M. H. Levelt Sengers and J. A. Given, Mol. Phys. 80, 899 (1993).

2. M. E. Fisher, J. Stat. Phys. 75, 1 (1994).

3. K. S. Pitzer, J. Phys. Chem. 99, 13070 (1995).

4. H. Weingärtner, M. Kleemeier, S. Wiegand, and W. Schröer, J. Stat. Phys. 78, 169 (1995).

5. G. Stell, J. Stat. Phys. 78, 197 (1995).

6. A. D. Kirshenbaum, J. A. Cahill, P. J. McGonigal, and A. V. Grosse, J. Inorg. Nucl. Chem. 24, 1287 (1962).

7. D. A. McQuarrie, J. Phys. Chem. 66, 1508 (1962).

8. H. L. Friedman, J. Phys. Chem. 66, 1595 (1962).

9. P. Debye and E. Hückel, Physik. Z. 24, 185 (1923). For a nice introduction see chapter XV of D. A. McQuarrie, Statistical Mechanics, Harper Collins publishers, New York, 1976).

10. K. S. Pitzer, M. C. P. de Lima, and D. R. Schreiber, J. Phys. Chem. 89, 1854 (1985).

11. R. R. Singh and K. S. Pitzer, J. Am. Chem. Soc. 110, 8723 (1988).

12. A. K. Arora et al., Phys. Rev. Lett. 60, 2438 (1988); B. V. R. Tata, M. Rajalakshmi, and A. K. Arora, Phys. Rev. Lett. 69, 3778 (1992); See also T. Palberg and M. Würth, Phys. Rev. Lett. 72, 786 (1994).

13. J.-P. Hansen and I. R. McDonald, Theory of Simple Liquids (Academic Press, London, 1986).

14. C. Caccamo, J. Chem. Phys. 91, 4902 (1989).

15. C. Caccamo and G. Malescio, J. Chem. Phys. 90, 1091 (1989).

16. P. U. Kenkare, C. K. Hall, and C. Caccamo, J. Chem. Phys. 103, 8111 (1995).

17. An exhaustive comparison between the results obtained here and in other works is not the goal of this article. It is however instructive to compare our predictions with the ones obtained by Kenkare et al. (Ref. 16 ). Although there is a general qualitative agreement between their phase diagrams and the ones presented here, there are clear quantitative discrepancies, better illustrated by the direct comparison of the location of the three-phase coexistence region obtained here and also in Ref. 16 . In their work, this region is found only at rescaled pressures $P^{*} \equiv T^{*} P a^{3} / k_{B} T<9.64 \times 10^{-5}$, where $a$ is the ionic size and $T^{*} \equiv a / \ell_{B}$ is the rescaled temperature $\left(\ell_{B}\right.$ is the Bjerrum length, cf. Section 2). For fixed $P^{*}=10^{-5}$, this triple phase region is found in Ref. 16 at $T^{*} \simeq 0.0657$ for both $\bar{Z}=2$ and 3 . For $\bar{Z}=2$ we find it at $T^{*} \simeq 0.1800$ and $P^{*} \simeq 10^{-3}$ and, for $\bar{Z}=3$, at $0.116 \lesssim T^{*} \lesssim 0.178$ and $2 \times 10^{-5} \lesssim P^{*} \lesssim 10^{-3}$. We expect a better agreement between the two approaches with the inclusion of higher order terms in Eq. (4).

18. Another example where a three-component ionic mixture was treated with the help of $\mathrm{HNC}$ can be found at B. Brami, J.-P. Hansen, and F. Joly, Physica A 95, 505 (1979). The system studied consists of a binary mixture of positively charged ions immersed in a neutralizing background of electrons with quantum corrections taken into account.
19. R. R. Netz and H. Orland, Europhys. Lett. 45, 726 (1999).

20. For a similar treatment as in [19], but applied to the OneComponent Plasma, see N. V. Brilliantov, Contrib. Plasma Phys. 38, 489 (1998). See also A. G. Moreira and R. R. Netz, Eur. Phys. J. D 8, 145 (2000).

21. See for instance H. B. Callen, Thermodynamics and an Introduction to Thermostatistics, 2nd ed. (John Wiley \& Sons, New York, 1985).

22. M. E. Fisher and Y. Levin, Phys. Rev. Lett. 71, 3826 (1993).

23. The influence of the ionic sizes and valences on the location of the critical point is discussed in some detail by A. K. Sabir, L. B. Bhuiyan and C. W. Outhwaite, Molec. Phys. 93, 405 (1998). See also I. Borukhov, D. Andelman and H. Orland, Phys. Rev. Lett. 79, 435 (1997).

24. A more rigorous way to include the hard-cores in the fieldtheoretic framework can be found at R. R. Netz and H. Orland, Eur. Phys. J. E 1, 67 (2000) (for symmetric mixtures) and A. G. Moreira and R. R. Netz, to be published (for asymmetric mixtures).

25. J. C. Rasaiah, D. N. Card, and J. P. Valleau, J. Chem. Phys. 56, 248 (1972).

26. One example where Debye-Hückel needs improvement is ionic pairing. One of the first attempts to treat this problem is due to Bjerrum - see N. Bjerrum, Kgl. Danske Videnskab. Selskab, Math.-fys. Medd. 7, 1 (1926); Selected Papers, pp. 108-119 (Einar Munksgaard, Copenhagen, 1949). For an instructive overview (although not up-todate) see R. A. Robinson and R. H. Stokes, Electrolyte Solutions (Butterworths, London, 1959). More recently, Fisher and Levin modified Bjerrum's theory and obtained good agreement with simulation results: see Ref. 22]. However, their approach is not consensual; for a critique and an alternative approach, see S. Yeh, Y. Zhou, and G. Stell, J. Phys. Chem. 100, 1415 (1996).

27. J. P. Valleau, J. Chem. Phys. 95, 584 (1991); G. Orkoulas and A. Z. Panagiotopoulos, J. Chem. Phys. 101, 1452 (1994); J. M. Caillol, D. Levesque, and J. J. Weis, J. Chem. Phys. 107, 1565 (1997) ; G. Orkoulas and A. Z. Panagiotopoulos, J. Chem. Phys. 110, 1581 (1999). 